\title{
IMPACTO DE LA INNOVACION SOBRE LA CONDUCTA EXPORTADORA EN EL SECTOR DE ALIMENTOS Y BEBIDAS DE COLOMBIA*
}

\author{
IMPACT OF INNOVATION ON EXPORT BEHAVIOR IN THE COLOMBIA'S \\ FOOD AND BEVERAGE INDUSTRY
}

\section{JOSE LUIS POLO OTERO**}

Universidad del Norte, Barranquilla, Colombia

\section{JOSE LUIS RAMOS RUIZ***}

Universidad del Norte, Barranquilla, Colombia

\section{AQUILES ANTONIO ARRIETA BARCASNEGRAS**** \\ Universidad del Norte, Barranquilla, Colombia}

\section{NATALIA RAMIREZ ARBELAEZ ${ }^{* * * *}$}

Universidad del Norte, Barranquilla, Colombia

\begin{abstract}
This paper tries to identify the impact of the different types of innovation on export behavior in the Colombian food and beverage industry. The application of the propensity score matching method reveals that only technological innovation has a significant effect on the sector's export behavior. In
\end{abstract}

* $\quad$ Este artículo es resultado del proyecto de investigación "Implementación de un programa de gestión de la innovación empresarial para fortalecer las Pymes de sectores estratégicos, Atlántico Caribe", financiado por el Fondo de Ciencia, Tecnología e Innovación del sistema general de Regalíasdepartamento del Atlántico.

** Profesor e investigador Instituto de Estudios Económicos del Caribe (IEEC), Departamento de Economía, Universidad del Norte, Barranquilla, Colombia. E-mail: lpoloj@ uninorte.edu.co. Dirección de correspondencia: Km.5 Vía Puerto Colombia, Área Metropolitana de Barranquilla, Colombia. Teléfono: (57) (5) 3509509 extensión: 4505.

*** Profesor e investigador Instituto de Estudios Económicos del Caribe (IEEC), Departamento de Economía, Universidad del Norte, Barranquilla, Colombia. E-mail: jramos@uninorte.edu.co. Dirección de correspondencia: Km.5 Vía Puerto Colombia, Área Metropolitana de Barranquilla, Colombia. Teléfono: (57) (5) 3509509 extensión: 4401.

**** Asistente de investigación Departamento de Economía, Universidad del Norte, Barranquilla, Colombia. E-mail: abarcasnegras@ uninorte.edu.co. Dirección de correspondencia: Km.5 Vía Puerto Colombia, Área Metropolitana de Barranquilla, Colombia.

***** Investigadora independiente. E-mail: arbelaezn@uninorte.edu.co. Dirección de correspondencia: Carrera 42 No 78-326, Barranquilla, Colombia. 
particular, product innovation impacts positively the internationalization of large companies, while process innovation does so in SMEs. The differences in strategic and innovative strategy explain the heterogeneity in the impacts obtained by the size of business and the type of innovation.

Keywords: Innovation, exports, propensity score matching, absorption capacity, $R \& D$, food and beverage, SMEs.

JEL Classification: O31, O32, F14, C21, L66.

\section{Resumen}

Este artículo busca identificar el impacto que tienen los distintos tipos de innovación respecto de la realización de exportaciones en la industria de alimentos y bebidas de Colombia. La aplicación del método de propensity score matching revela que solo la innovación tecnológica tiene un efecto significativo en la conducta exportadora del sector. Particularmente, la innovación en producto impacta positivamente la internacionalización de las empresas grandes, mientras que la innovación en procesos lo hace en las Pymes. Diferencias en la orientación estratégica e innovadora explican la heterogeneidad presente en los impactos obtenidos según tamaño empresarial y tipo de innovación.

Palabras clave: Innovación, exportaciones, propensity score matching, capacidad de absorción, $I+D$, alimentos y bebidas, Pymes.

Clasificación JEL: O31, O32, F14, C21, L66.

\section{INTRODUCCION}

El sector de alimentos y bebidas juega un rol importante dentro de la estructura industrial de Colombia. De acuerdo con los datos de la Encuesta Anual Manufacturera (EAM) adelantada por el Departamento Administrativo Nacional de Estadística (DANE), esta actividad en 2015 aglutinó el 24,4\% del personal ocupado, generó el 30,3\% de la producción bruta y creó el 31,8\% del valor agregado industrial. La dimensión de la importancia del sector llega a ser más grande en cuanto es una actividad productiva constituida en su mayoría por Pymes. Por tanto, resulta trascendental que las empresas de este sector incrementen su competitividad, de forma que puedan seguir contribuyendo al crecimiento económico del país. 
La entrada de estas firmas al mercado internacional junto con el incremento del flujo de actividades comerciales supone la implementación de diversas estrategias, dentro de las que destacan las actividades de innovación como una de las herramientas clave para este fin. En la medida que los recursos de innovación fomentan mejoras en varios aspectos de la productividad que a su vez inciden positivamente en varios resultados empresariales, ente ellos la internacionalización, la realización de proyectos de innovación presenta alternativas para encontrar nuevos mercados y oportunidades de aumentar los volúmenes de producción. De esta forma, las exportaciones y la innovación entran en una dinámica que genera sinergias positivas para el sector de interés.

Por ello, el presente trabajo tiene como objetivo establecer el impacto que tiene la realización de los diferentes tipos de innovación (producto, proceso, marketing y organizacional) en la conducta exportadora de las empresas del sector de alimentos y bebidas en Colombia. Para ello se recurre a la metodología del propensity score matching como técnica de estimación empírica.

Las principales contribuciones de la investigación apuntan a dos frentes. Por una parte, aportar criterios al empresariado del sector y a los encargados de la política pública nacional acerca de la forma en que deben orientar y guiar los recursos destinados a la innovación, de manera que los resultados obtenidos se traduzcan en mayor competitividad. Por otro lado, este trabajo integra un análisis que involucra los cuatro tipos de innovación, no solo la innovación tecnológica como acostumbra el grueso de la literatura concerniente al tema.

El documento se estructura en 7 secciones, constituyendo esta introducción la primera. La segunda corresponde a una revisión del estado del arte respecto de la relación entre exportaciones e innovación. Posteriormente se ofrece un marco teórico para explicar este vínculo. Una descripción de la innovación en el sector de alimentos y bebidas de Colombia constituye la cuarta sección. En la quinta se explica la metodología del propensity score matching y se describen los resultados obtenidos tras su aplicación para el tema de estudio. Luego se presenta un análisis de los hallazgos. Finalmente se hacen recomendaciones de política y se concluye.

\section{REVISION DE LITERATURA}

A lo largo de los años, varios estudios han tratado la relación entre innovación y exportaciones a nivel de la firma. Tradicionalmente, el análisis se concentra en el contenido empírico, así como en países desarrollados y en aquellos que han aumentado su participación en el comercio mundial en el último tiempo. Generalmente estas investigaciones apuntan a profundizar y comprender acerca de los retornos económicos de la innovación tecnológica, asociados a la introducción de productos y procesos nuevos o mejorados al mercado, al tiempo que se enfocan de forma casi exclusiva en los gastos en I+D. 
Bajo este enfoque, la literatura acerca del análisis de las variables de innovación en las empresas y la relación que existe con su conducta exportadora incluye un conjunto de factores que soportan este vínculo. La conducta exportadora se ha estudiado en los trabajos empíricos como resultado de modelos que incluyen un conjunto de atributos estructurales, conductuales y de desempeño de la empresa que tienen alguna influencia y peso en la decisión exportadora. En estos estudios se toma como unidad de análisis a la empresa y se evalúan las condiciones que la llevan a tomar la decisión de innovar, de tal forma que se recogen las ganancias por implementar actividades tecnológicas y desarrollar nuevos productos o procesos (Wakelin, 1998). Las investigaciones existentes demuestran que el crecimiento, e incluso la supervivencia de las empresas, dependen de la productividad y la innovación, que actúan como facilitadores del acceso a nuevos mercados (Martins, Gómez-Araujo y Vaillant, 2014).

Márquez-Ramos, Martínez-Zarzoso y Suárez-Burguet (2011) establecen que los países con mayor inversión en I+D y catalogados como líderes tecnológicos mantienen una relación positiva entre la innovación tecnológica y las exportaciones, ya que el efecto es siempre positivo y se magnifica por las mejoras tecnológicas. Adicionalmente, concluyen que el efecto de la innovación tecnológica en el comercio puede variar de acuerdo con el logro tecnológico mediante la generación de una relación no lineal y que, en búsqueda de fomentar las exportaciones, los países tienen que considerar no solo las capacidades de adquisición y asimilación de conocimiento, sino también las capacidades de transformación y explotación una vez que se ha alcanzado un nivel mínimo de capacidad de absorción potencial.

De manera similar, Roper y Love (2002) analizan los factores tecnológicos, considerando proxies de tecnología, los cuales, en combinación con la estructura de mercado, registran el mayor impacto en la determinación de las exportaciones manufactureras a largo plazo, en comparación con la competitividad-precio. Los autores plantean que mantener inversiones en $\mathrm{I}+\mathrm{D}$ durante largo tiempo, obtener patentes sistemáticamente, adquirir tecnología desde el extranjero y, en general, realizar innovaciones de proceso o producto, conducen en el largo plazo a mayores exportaciones. Esta idea se ve reforzada por quienes consideran que cuanto mayor sea la inversión en innovación tecnológica realizada por la empresa mayor será su nivel de desempeño exportador, ya que las capacidades tecnológicas de producción incluyen no solo las habilidades con que determinadas tecnologías se generan y mejoran en la firma, sino también de qué manera se utilizan los esfuerzos internos para asimilar las tecnologías compradas o imitadas de otras empresas (Peris, Mestre, y Zornoza 2004).

En el caso de Latinoamérica, la evidencia encontrada, aunque escasa, indica la existencia de un vínculo positivo entre innovación y éxito exportador. Las empresas que llevan sus productos al extranjero realizan más esfuerzos en actividades de innovación, obtienen mayores y más relevantes resultados empresariales y desarrollan mayores competencias tecnológicas (Milesi y Aggio, 2008). En efecto, estudios enfocados en analizar los determinantes del éxito exportador en Pymes de Argentina (Moori Koeni. Milesi y Yoguel, 2001), Chile (Moori Koenig et al., 2004) y Colombia 
(Moori Koenig et al., 2005), han estudiado numerosas dimensiones, entre las que se encuentran las vinculadas a competencias tecnológicas y comerciales, logrando apreciar un impacto positivo de las asociadas a la innovación respecto de la conducta exportadora. Asimismo, Estrada y Heijs (2003) encontraron en su investigación aplicada a un grupo de Pymes en México que la asignación de recursos económicos a I+D, la adquisición de nuevas tecnologías, la presencia de personal calificado y la compra de bienes para introducción de nuevos procesos o productos, incrementa potencialmente la capacidad exportadora de las empresas.

Con el fin de identificar cuál de los dos tipos de innovación tecnológica tiene mayor efecto en las exportaciones, algunos trabajos logran priorizar el rol de alguna de ellas en el proceso de internacionalización empresarial. Acerca del particular, para Palangkaraya (2012) el desarrollo de nuevos bienes está directamente ligado con la salida al mercado internacional de una empresa por primera vez. En su estudio encuentra evidencia que innovar en producto en un periodo conduce a una mayor probabilidad de convertirse en nuevo exportador en ese mismo lapso de tiempo.

Por su parte, Nassimbeni (2001) plantea que la gestión de actividades relacionadas con los productos parece ser uno de los factores fundamentales para que las pequeñas empresas entren en mercados internacionales. La inversión en tecnología, y más exactamente la innovación en procesos, juega un papel secundario en relación con la innovación de producto, concluyendo que la simple posesión de un nivel tecnológico elevado no garantiza una ventaja efectiva para exportar. Este planteamiento es soportado por Eggertsson (1995), quien encuentra que la propensión de las empresas a exportar está estrictamente ligada a su capacidad de innovar en producto mientras que está menos relacionada con el perfil de procesos (fabricación, control de calidad, gestión, diseño, comunicación, manipulación, tecnologías de almacenamiento, etc.) de la empresa.

Una orientación similar propone Bocquet y Musso (2010), quienes examinan el impacto de la innovación de productos en el comportamiento exportador de las empresas manufactureras francesas y encuentran que la innovación de este tipo es el principal factor para promover las exportaciones, tanto en términos de participación como de intensidad, mientras que no existe tal evidencia para la innovación de procesos. Los resultados también muestran que la participación de la innovación en productos aumenta significativamente la probabilidad de comenzar a exportar. Estos hallazgos están alineados con modelos teóricos recientes como los modelos de heterogeneidad de firmas en dos dimensiones (Hallak y Sivadasan, 2009) y los modelos de empresas multiproducto (Nocke y Yeaple, 2006), los que proporcionan una caracterización más matizada del proceso de innovación.

Otros estudios llegan a la conclusión que el gasto en I+D no aumenta la probabilidad de exportar solo por la capacidad de producir nuevos bienes, sino que va más ligado a la mejora que esto trae atinente a la adquisición de conocimiento, importante para superar las barreras y obstáculos al introducirse en mercados internacionales (Harris y Moffat, 2011). Entretanto, trabajos como Van Beveren y Vandenbussche (2009) 
sugieren que la combinación de innovación de productos y procesos, más que cualquiera de las dos por separado, es lo que impulsa a las empresas al mercado internacional.

Un tema importante en este punto tiene que ver con la causalidad de la relación entre innovación y conducta exportadora. Mientras en los trabajos citados el efecto va desde innovación hacia internacionalización, Bernard y Jensen (1999) y Milesi y Aggio (2008) sugieren que la exigencia derivada del nivel competitivo en los mercados internacionales hace que las exportaciones sean un determinante de la innovación empresarial. Precisamente, Bernard y Jensen (1999) plantean que la discusión típica se centra en la relación causal entre el éxito de la empresa y la exportación de las firmas, dejando de lado el argumento que la exportación causa un mejor rendimiento de la empresa. Para los autores, bajo este último escenario el enfoque se concentra en la naturaleza prospectiva de las empresas, las que reconocen que una vía potencial de crecimiento continuo para sus productos se da por medio de las ventas externas. Estudiando la interacción entre la internacionalización y el rendimiento de la empresa, antes, durante y después de exportar, los resultados dan cuenta que el éxito y los nuevos productos conducen a la exportación. No obstante, desde la perspectiva de los beneficios de vender al extranjero, estos se vuelven menos claros con el tiempo, lo que sugiere que es poco probable que las empresas que ingresan al mercado internacional aumenten sustancialmente su productividad.

Siguiendo la orientación que explora el efecto que va desde exportaciones hacia actividades de innovación, Crespi y Zúñiga (2012) en su estudio concerniente a los determinantes de la innovación tecnológica y su impacto en la productividad del trabajo de los países latinoamericanos reportan que la realización de exportaciones aumenta la propensión a invertir en actividades de innovación y alienta la inversión en innovación en Argentina, Chile y Colombia. Asimismo, se valida que las empresas que innovan tienen mayor productividad laboral que aquellas que no lo hacen.

Este trabajo pone de manifiesto que, para los países en desarrollo, el impacto de las exportaciones respecto de la innovación depende de otros factores, así es el caso de la magnitud de las restricciones financieras. Cuando estas son laxas, y por tanto las empresas tienen acceso a la financiación, ambas actividades pueden trabajar en forma complementaria (reforzándose entre sí) (Gorodnichenko y Schnitzer, 2010).

\section{ELEMENTOS CONCEPTUALES PARA EXPLICAR EL FENOMENO DE ESTUDIO}

La literatura conceptual pertinente a la relación existente entre los diferentes tipos de innovación y las exportaciones ha sido tratada a lo largo de los años, en la búsqueda de explorar la incidencia del proceso innovador en el aumento de la productividad y competitividad de las empresas. En la actualidad, la internacionalización se ha convertido en una necesidad para las organizaciones. Según Johanson y Vahlne (2006), para que una empresa acceda a mercados extranjeros, en primer lugar, se debe desarrollar en 
su mercado nacional; seguido de esto, debe llevar a cabo exportaciones irregulares; posteriormente, debe emplear agentes independientes e implantar filiales comerciales; y por último, poner en marcha filiales productivas.

Los factores que motivan este proceso están relacionados con la búsqueda de nichos de mercados no ocupados en el extranjero y clientes potenciales a los cuales dirigirse; la comercialización y distribución; y finalmente los procesos de innovación tecnológica, para ello algunas empresas se soportan en inversiones en I+D, mientras otras lo hacen por medio de la modernización tecnológica, en busca de elementos diferenciadores que reduzcan costos e incrementen la eficiencia y productividad (Pedrero, 2014).

De acuerdo con la teoría evolutiva de internacionalización de las empresas, las dificultades de negociación con intermediarios, sumadas a los altos costos y competencia en mercados internos, obligan al innovador a llevar su experiencia productiva hacia territorios nuevos (Alonso, 1994). Esta externalización de la inversión deriva al mismo tiempo en transferencia de conocimiento hacia economías receptoras y una difusión constante de la producción que aumenta la probabilidad de exportación de las firmas hacia otros países.

Tradicionalmente, la literatura referida a comercio exterior identifica dos posturas teóricas para dar razón a la relación entre innovación y exportaciones. Por una parte, el impacto positivo de la primera sobre la segunda se explica por un mecanismo de selección, mediante este las empresas más productivas entran en el mercado de exportación y esto da lugar a la posibilidad de que los exportadores aprendan de sus contactos en el extranjero, adopten nuevas tecnologías de producción y aumenten la productividad y el rendimiento de la empresa frente a la competencia (Salomon y Shaver, 2005). Por otro lado, la hipótesis de learning by exporting establece una relación causa-efecto desde la internacionalización hacia la innovación, en respuesta a la experiencia que adquieren las empresas por el conocimiento proveniente del extranjero y la tecnología del mercado global (Grossman y Helpman, 1993; Aghion et al., 1998; Salomon y Shaver, 2005).

La evidencia muestra mayor apoyo a la hipótesis de selección debida al vínculo entre productividad y exportaciones. Trabajos como Aw, Chen y Roberts (2001); Delgado, Farinas y Ruano (2002) y Greenaway y Kneller (2007) destacan que, en general, las empresas que exportan tienen un nivel de productividad superior al de las que no lo hacen, previo al comienzo de las actividades de venta en el exterior. Acerca del particular, Cassiman, Golovko y Martínez (2010) afirman que las empresas con pocas ventajas comparativas y baja productividad saldrán del mercado, mientras que las empresas con suerte y alta productividad continuarían prosperando y creciendo.

Precisamente la heterogeneidad en productividad entre firmas es el centro de análisis de la mayoría de investigaciones relacionadas con el tema. Sobre la base del trabajo acerca de dinámica industrial de Jovanovic (1982), los modelos teóricos de Bernard et al., (2003), Melitz (2003), y Yeaple (2005) asumen una distribución de productividad exógena entre firmas, sin llegar a explicar el porqué de las diferencias. 
Otra corriente de literatura busca endogeneizar la heterogeneidad en productividad de las empresas, partiendo del supuesto que estas deciden invertir en actividades que aumenten su productividad antes de exportar. Una fuente importante para justificar las diferencias en productividad viene de las inversiones en I+D y actividades de innovación. Dentro de estas estructuras teóricas se tiene a Costantini y Melitz (2008), quienes estudian el nexo entre la inversión en innovación y exportaciones en el marco de liberalización de los regímenes de comercio, relacionando la innovación como un shock en la productividad futura de la empresa. Bustos (2011) desarrolla un modelo similar para estudiar la presencia de una asociación entre la actualización tecnológica y el mercado internacional, en las empresas argentinas tras el ingreso del país a MERCOSUR.

Asimismo, modelos como el de Aw, Roberts y Yi Xu (2011) reconocen la posibilidad que existan conexiones intertemporales entre las exportaciones, productividad e innovación. Bajo este modelo estructural dinámico se permite que la decisión de un productor de invertir en I+D y exportar afecte de forma endógena la trayectoria futura de la productividad, de tal forma que existe una doble vinculación de las variables. En primer lugar, el retorno procedente a cada elección óptima de inversión en I+D y exportación aumenta con la productividad subyacente del productor, lo que lleva a los productores de alta productividad a autoseleccionarse en ambas actividades de inversión. En segundo lugar, cada inversión afecta directamente a la productividad futura, que actúa para reforzar el efecto de selección. En este sentido, la autoselección de empresas de alta productividad es el canal dominante que impulsa la participación en el mercado de exportación y el gasto en $\mathrm{I}+\mathrm{D}$, lo que se refuerza por el efecto de cada inversión en la productividad futura.

Dentro de los argumentos expuestos para explicar la conexión entre I+D/ innovación y conducta exportadora se reconoce el papel de la capacidad tecnológica para hacer más competitiva a las firmas, lo que a su vez les permite invertir en I+D y mejorar su capacidad de innovación (Rodil, Vence y Sánchez, 2015). También se destaca la ventaja que supone la internacionalización en cuanto expande las ventas de las empresas, facilitándole repartir los costos de la innovación, normalmente fijos, en un mayor volumen de demanda (Álvarez y Robertson, 2004). Adicionalmente, la inversión en $\mathrm{I}+\mathrm{D}$ fortalece los activos de conocimiento contribuyendo al incremento de la capacidad de las empresas para asimilar el conocimiento externo.

Otra perspectiva teórica para entender la conexión entre innovación y exportaciones proviene de la teoría basada en los recursos, la que reconoce la heterogeneidad entre firmas a partir de la consideración de un conjunto de recursos productivos, caracterizados por su especificidad y su condición de inimitables por otra firma. Las investigaciones tras la publicación de Penrose (1959) han propuesto una teoría de ventaja competitiva, según esta, aparece en las empresas en la medida que se hagan presentes recursos con características distintivas. Estos recursos deben ser imposibles de sustituir, escasos, imperfectamente imitables y facilitar la implementación de estrategias para incrementar su eficiencia (Barney, 2001). Aquellas empresas con recursos de este tipo gozarán de 
una ventaja competitiva potencial que facilitará su ingreso al mercado internacional (Roper y Love, 2002; López y García, 2005).

En este sentido, los activos intangibles basados en la información y el conocimiento, y sobre todo los tecnológicos, son reconocidos como el sustento de las ventajas competitivas de las empresas, en tanto son los recursos que mejor se adecuan a las características señaladas. Debido a que la innovación se soporta tradicionalmente en este tipo de recursos, puede ser vista como un proceso que lidera a la mejora de procesos y a la introducción de nuevos productos, lo que en conjunto con la globalización, la segmentación de mercados y los productos personalizados abren la puerta para una mayor competitividad vía exportaciones (López y García, 2005). Desde esta concepción, se reitera que la capacidad tecnológica y de innovación es un elemento que fomenta la internacionalización, al tiempo que brinda la opción a las empresas de usar el conocimiento en el entorno internacional.

\section{DESCRIPCION DE LA INNOVACION EN EL SECTOR DE ALIMENTOS Y BEBIDAS DE COLOMBIA}

Previo al análisis empírico, se presenta una mirada descriptiva de la innovación en la industria colombiana de producción de alimentos y bebidas. Para ello se presentan estadísticas descriptivas de la innovación en las empresas del sector y se comparan con el resto de empresas industriales del país. La información procede de la Encuesta de Desarrollo e Innovación Tecnológica en la industria manufacturera -EDIT VII-, elaborada por el DANE para los años 2013 y 2014. Esta encuesta investigó las conductas de innovación de 8.835 empresas, de estas, 18,05\% tienen como actividad principal la producción de alimentos y bebidas. Dentro de este sector, como se indicó en la introducción, el grueso de las empresas son Pymes ${ }^{1}$, representando aproximadamente $68 \%$ del total.

La Tabla 1 indica el porcentaje de empresas de alimentos y bebidas que realizó cada uno de los tipos de innovación, considerando el tamaño de las empresas en la muestra. Tanto para el caso de las Pymes como de las empresas grandes en el sector, se observa que el tipo de innovación que acumula la mayor proporción de compañías es la innovación en proceso.

Comparando entre las pequeñas y medianas empresas, se percibe que la industria de alimentos y bebidas tiene una mayor proporción de compañías realizando cada uno de los tipos de innovación, a excepción de las innovaciones en producto, que el resto de actividades industriales en su conjunto.

1 En este trabajo se reconoce una Pyme como aquella empresa que cumple con la disposición establecida en el Estado colombiano por la Ley 590 de 2.000 en el artículo 2. En este sentido, en la categoría de Pymes se ubican todas las empresas que tengan entre 11 y 200 empleados o posean activos totales por un valor que oscile entre 501 y 15.000 salarios mínimos mensuales legales vigentes. 


\section{TABLA 1}

\section{PORCENTAJE DE EMPRESAS DE ALIMENTOS Y BEBIDAS QUE REALIZO CADA TIPO DE INNOVACION, SEGUN TAMAÑO}

\begin{tabular}{|l|c|c|c|c|c|c|c|c|c|c|c|c|}
\hline \multirow{2}{*}{ Sector económico } & \multicolumn{3}{|c|}{ Producto } & \multicolumn{3}{c|}{ Proceso } & \multicolumn{3}{c|}{ Organizacional } & \multicolumn{3}{c|}{ Marketing } \\
\cline { 2 - 13 } & Total & Pyme & Grande & Total & Pyme & Grande & Total & Pyme & Grande & Total & Pyme & Grande \\
\hline Alimentos y bebidas & 8,7 & 9,1 & 34,7 & 13,4 & 11,8 & 36,7 & 6,0 & 5,4 & 14,9 & 5,7 & 4,7 & 17,3 \\
Resto de actividades & 9,4 & 9,5 & 29,6 & 10,2 & 10,4 & 31,1 & 4,9 & 4,9 & 15,9 & 3,4 & 3,3 & 10,2 \\
\hline
\end{tabular}

Fuente: EDIT-VII.

Esta situación se sostiene para las empresas de gran tamaño, exceptuando la innovación de tipo organizacional.

Otra característica de las empresas dedicadas a la producción de alimentos y bebidas es la condición incremental de la mayoría de sus innovaciones. De acuerdo con los datos suministrados por la EDIT, 99,7\% de las innovaciones que se dan en el sector son incrementales. Este comportamiento es, sin embargo, común en la industria manufacturera del país, donde para el resto de sectores este porcentaje alcanza 99,4\%.

Finalmente, al estudiar el número de innovaciones promedio que obtiene una empresa innovadora en el periodo 2013-2014, la Tabla 2 revela que el sector de alimentos y bebidas colombiano registra una cantidad considerablemente alta en comparación con otros renglones industriales de importancia para el país, siendo superada solo por los sectores de producción de químicos y fabricación de textiles.

TABLA 2

NUMERO PROMEDIO DE INNOVACIONES POR EMPRESA INNOVADORA

\begin{tabular}{|l|c|}
\hline Sector económico & $\begin{array}{c}\text { Número promedio de innovaciones } \\
\text { por empresa innovadora }\end{array}$ \\
\hline Alimentos y bebidas & 1,74 \\
Producción de textiles & 1,78 \\
Producción de papel y cartón & 1,49 \\
Fabricación de productos de plástico & 1,45 \\
Fabricación de maquinaria y equipo & 1,31 \\
Fabricación de productos químicos & 2,88 \\
Fabricación de productos farmacéuticos & 1,59 \\
\hline
\end{tabular}

Fuente: EDIT-VII. 


\section{ENFOQUE EMPIRICO: METODO DE EMPAREJAMIENTO}

Para determinar el impacto que tiene la innovación respecto de la condición exportadora de las empresas del sector de alimentos y bebidas en Colombia, se recurre a técnicas aplicadas en la evaluación de impacto. De forma específica, se hace uso del método de emparejamiento o propensity score matching (PSM). Esta metodología permite establecer el vínculo causal entre la participación en un programa o proyecto y una(s) variable(s) de resultado. En el caso de este trabajo se considera como "programa o proyecto" aquella situación en la que una empresa realiza uno de los cuatro tipos de innovación definidos por el Manual de Oslo (OECD, 2005), a saber: innovación en producto, innovación en proceso, innovación organizacional e innovación en marketing. Por su parte, se toma como variable de resultado el hecho de adelantar ventas al extranjero o no. De forma tal, que se evalúa el efecto de cada una de las cuatro formas de innovar sobre la ejecución de actividades de exportación.

Para cada tipo de innovación, su realización define un conjunto de empresas que participan en el "programa o proyecto", conocido como grupo de tratamiento. Aquellas firmas que no hacen parte del tratamiento, referidas como control, se utilizan como input para la construcción de un escenario contrafactual, el que refleja la situación experimentada por los tratados, en cuanto a la variable de resultado, si no hubieran adelantado la innovación respectiva.

Bajo estas definiciones, la participación en un proyecto de innovación no es aleatoria. La presencia de características, observables y no observables, que provoca que unas empresas decidan innovar en una forma específica, y otras no, posiblemente también sea causante de diferencias en la variable de resultado. Por tanto, es posible que la realización de exportaciones del grupo de tratamiento y la realización de exportaciones por parte del grupo de control sean diferentes, aun si el desarrollo de cualquier tipo de innovación no existiera. Este hecho se conoce como sesgo de selección. Empíricamente, este problema es resuelto mediante el método de emparejamiento.

PSM es una técnica semiparámetrica que empareja a cada miembro del grupo de tratamiento con al menos un miembro del grupo de control, sobre la base del supuesto de independencia condicional, el que establece que la participación en un proyecto se soporta únicamente en las variables observadas (recogidas en un vector $X$ ). De acuerdo con Bernal y Peña (2011), la versión más simple de esta metodología radica en encontrar un "clon" en el grupo de control para cada miembro del grupo de tratamiento. La alternativa aplicada en PSM compara aquellos casos cercanos, en términos de $X$, de tal forma que las unidades tratadas son emparejadas con las no tratadas a partir de la probabilidad predicha de participar en el programa o propensity score, $P(X)$, lo que supone que PSM se calcula exclusivamente en la región de soporte común (SC). Dicha condición implica que en la estimación solo se consideran aquellas observaciones (empresas) del grupo de control que tengan probabilidades de participación $P(X)$ semejantes a las probabilidades del conjunto de tratamiento. 
Bajo el cumplimiento de los supuestos de independencia condicional y soporte común, el método de emparejamiento estima el efecto promedio del tratamiento (ATT, por sus siglas en inglés), que para el contexto analizado es definido como la diferencia en el promedio de la variable de resultado (EXPORTACIÓN) entre aquellos que han sido tratados $(T=1)$-por ejemplo aquellas empresas que han realizado cualquiera de los tipos de innovación-y aquellos que no lo han sido $(T=0)$.

$$
\tau_{A T T}^{P S M}=E_{P(X) \mid T=1}\{E[\text { EXPORTACIÓN } \mid T=1, P(X)]-E[\text { EXPORTACIÓN } \mid T=0, P(X)]\}
$$

Donde $E_{P(X) \mid T=1}$ corresponde al valor esperado con relación a $P(X)$, condicional en realizar un proyecto de innovación y $T$ a la variable de tratamiento.

De tal forma que, condicionado respecto de la probabilidad de participación, se genera una estimación insesgada y consistente (Rosenbaum y Rubin, 1983).

\subsection{Estimación PSM}

Así como se comentó, mediante la aplicación del método de emparejamiento se busca establecer el efecto que tiene la realización de cada tipo de innovación acerca del hecho de exportar o no. El primer paso consiste en la estimación de la probabilidad de cada empresa de adelantar cada tipo de innovación, para ello se hace uso de la técnica de probit mutivariado, permitiendo examinar la correlación de los errores en las cuatro ecuaciones, una para cada tipo de innovación, lo que parece razonable si existen shocks que pueden afectar a los cuatro tipos de innovación.

En cada ecuación se regresa una variable dicótoma, con valor de 1 si la empresa realizó la innovación respectiva, sobre un conjunto de variables observables que determinan la ejecución de dicho proyecto de innovación. La identificación teórica inicial de las variables que inciden en cada tipo de innovación se realizó en función de los resultados presentados en investigaciones previas. La Tabla 3 muestra la revisión adelantada, señalando con un signo más (+) aquellas variables que inciden en cada tipo de innovación, así como la forma de medición de cada una en este trabajo.

Los datos empleados se obtuvieron de la Encuesta de Desarrollo e Innovación Tecnológica en la industria manufacturera -EDIT VII-. Se hicieron estimaciones para el total de empresas del sector de alimentos y bebidas, y para la muestra fragmentada entre empresas grandes y Pymes.

Para una estimación apropiada del ATT se consideró que debido a que el emparejamiento no se adelanta sobre el total de variables observables, sino solo sobre la probabilidad de participación, la condición de balanceo debe ser satisfecha. Es decir, que tanto la probabilidad promedio de participación como la media de las variables en el vector $X$ deben ser idénticas entre los grupos de tratamiento y control.

Esta condición no es cumplida por el vector de variables $X$ definido por medio de la revisión teórica para cada tipo de innovación. Por tanto, en la estimación definitiva 


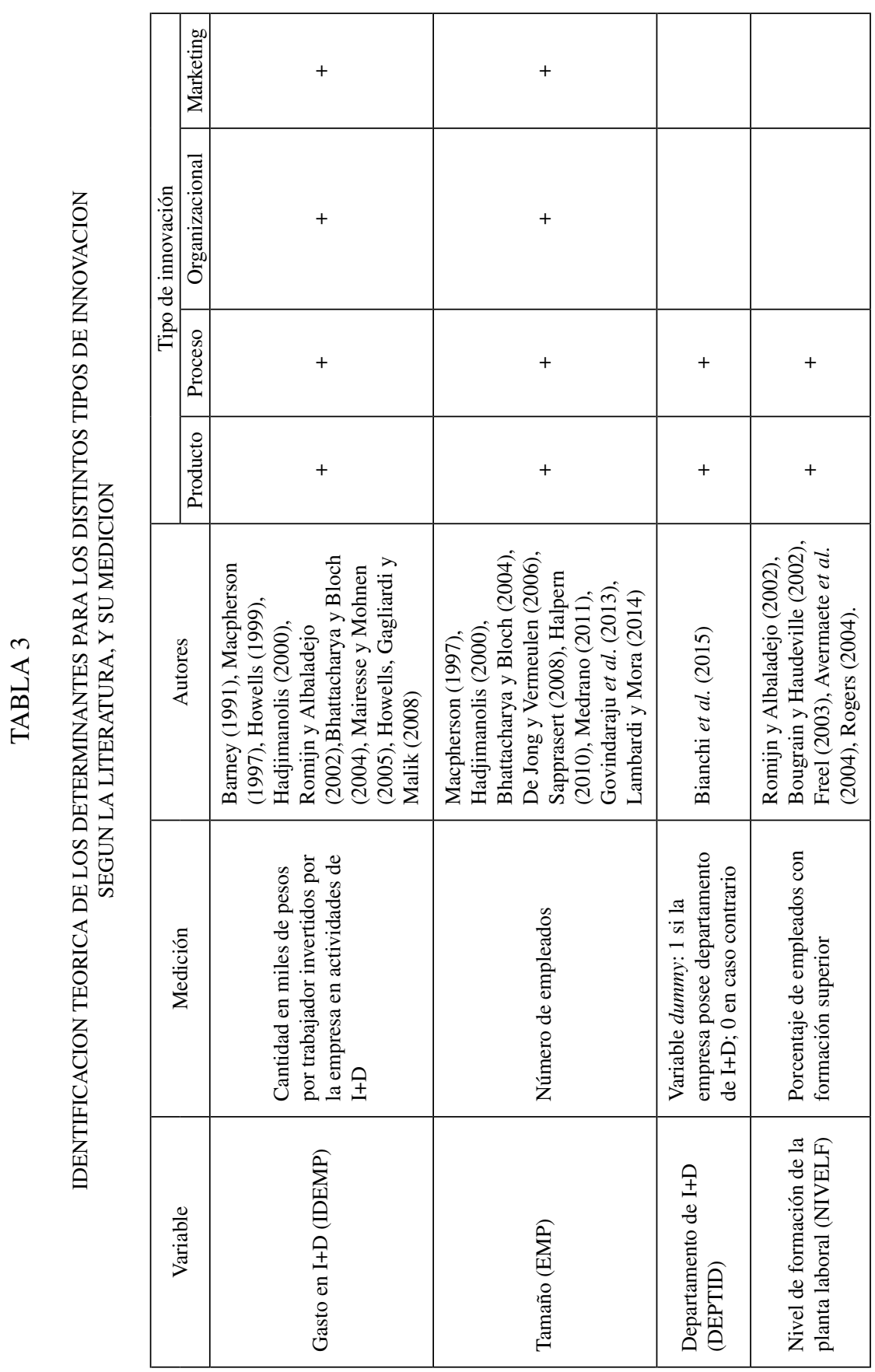




\begin{tabular}{|c|c|c|c|c|c|}
\hline 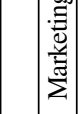 & & + & + & + & + \\
\hline 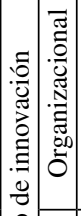 & + & + & & + & \\
\hline 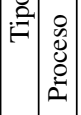 & + & + & & + & \\
\hline $\mid \begin{array}{l}0 \\
0 \\
0 \\
0 \\
0 \\
0 \\
0\end{array}$ & + & + & & + & \\
\hline 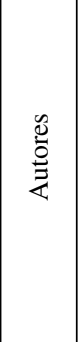 & 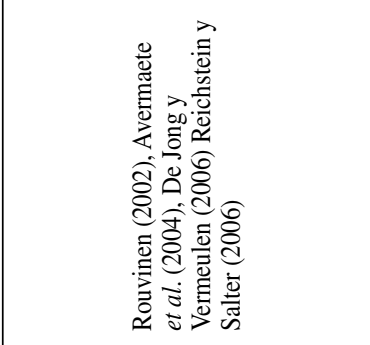 & 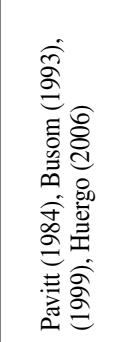 & 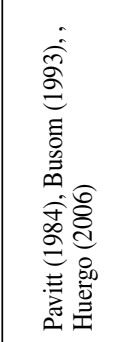 & 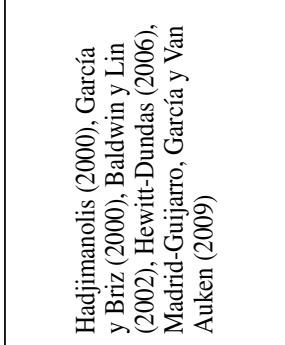 & 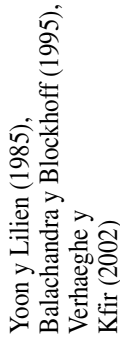 \\
\hline $\begin{array}{l}\frac{\pi}{0} \\
: \frac{0}{0} \\
\sum^{0}\end{array}$ & 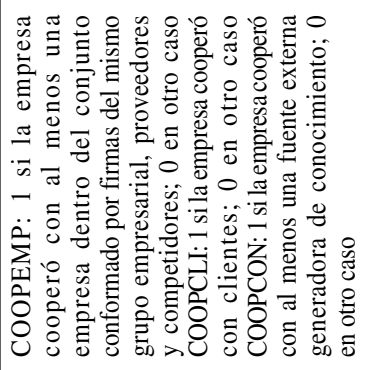 & 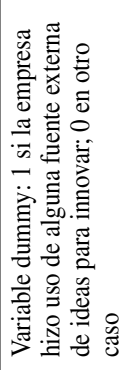 & 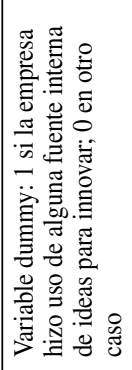 & 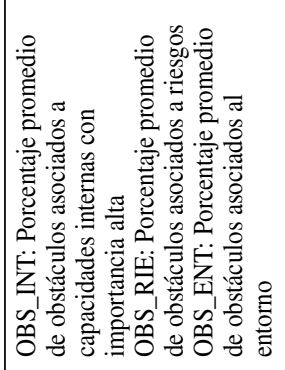 & 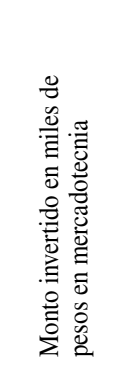 \\
\hline 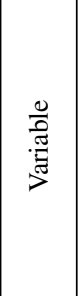 & 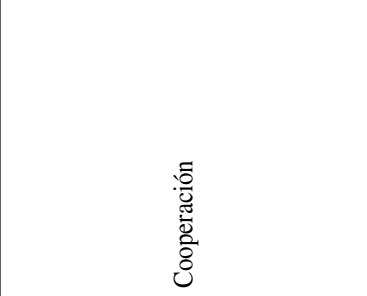 & 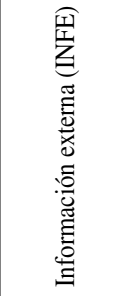 & 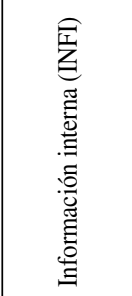 & 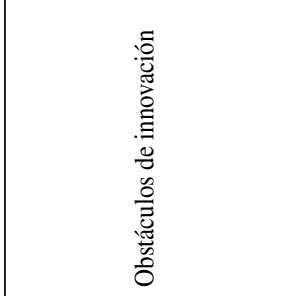 & 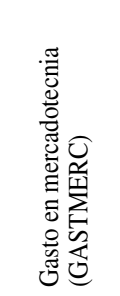 \\
\hline
\end{tabular}


de las probabilidades de innovar y propensity score solo se incluyeron las variables que garantizan la condición de balanceo y en su mayoría resultan significativas. El cambio propuesto en la especificación no altera significativamente el poder explicativo de los modelos ni las estadísticas de clasificación.

Las Tablas 4, 5 y 6 presentan los resultados para el total de la muestra, para las Pymes y para las empresas grandes, respectivamente ${ }^{2}$. En cada caso se restringió la muestra a la región de soporte común.

\section{TABLA 4}

ESTIMACION PROBIT MULTIVARIADO TOTAL EMPRESAS DE ALIMENTOS Y BEBIDAS

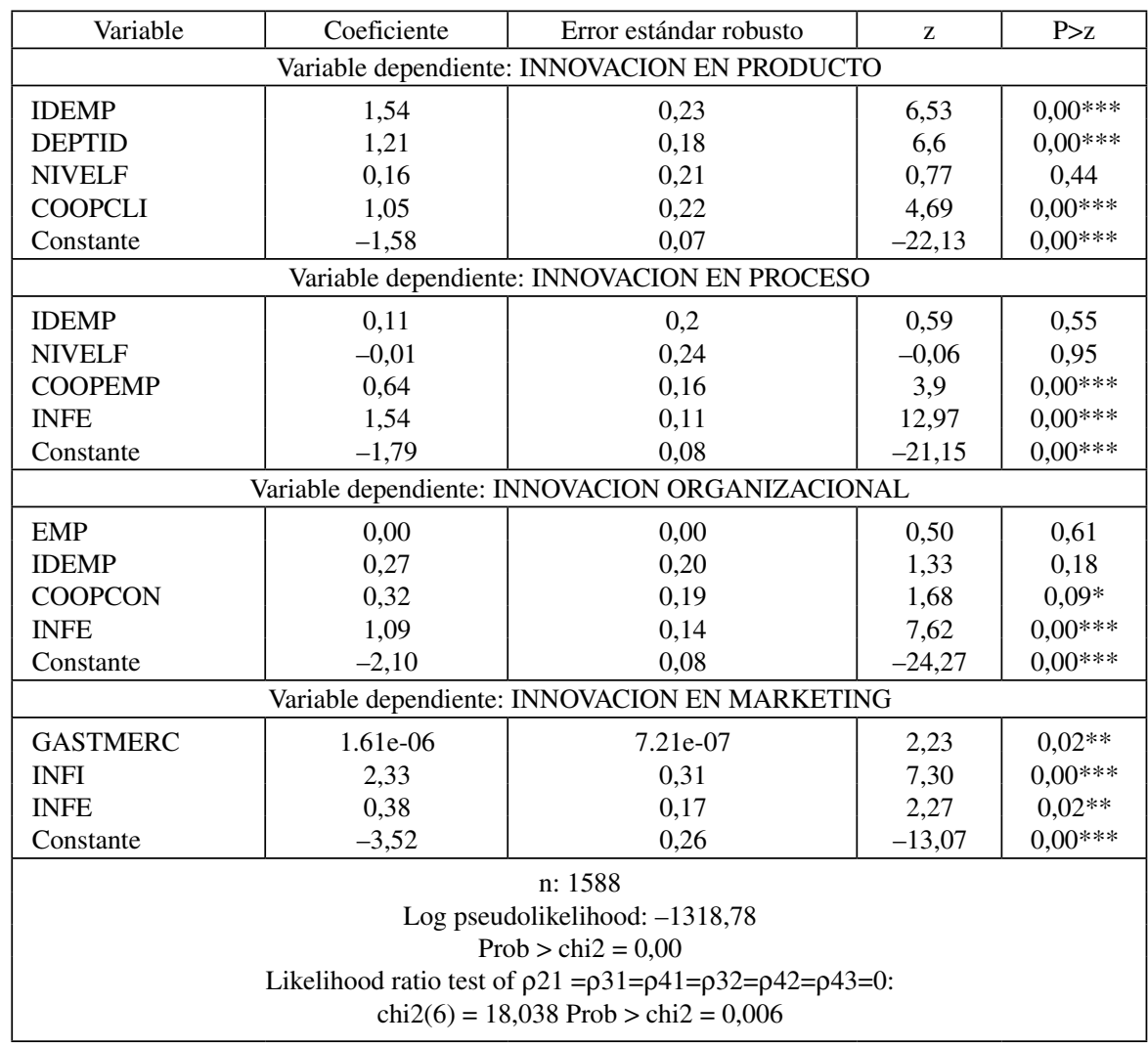

Significancia: $* * * 1 \%, * * 5 \% ; * 10 \%$.

2 Como anexo se presentan las estadísticas descriptivas de las variables consideradas en cada ecuación en cada modelo probit multivariado. 
TABLA 5

ESTIMACION PROBIT MULTIVARIADO PYMES ALIMENTOS Y BEBIDAS

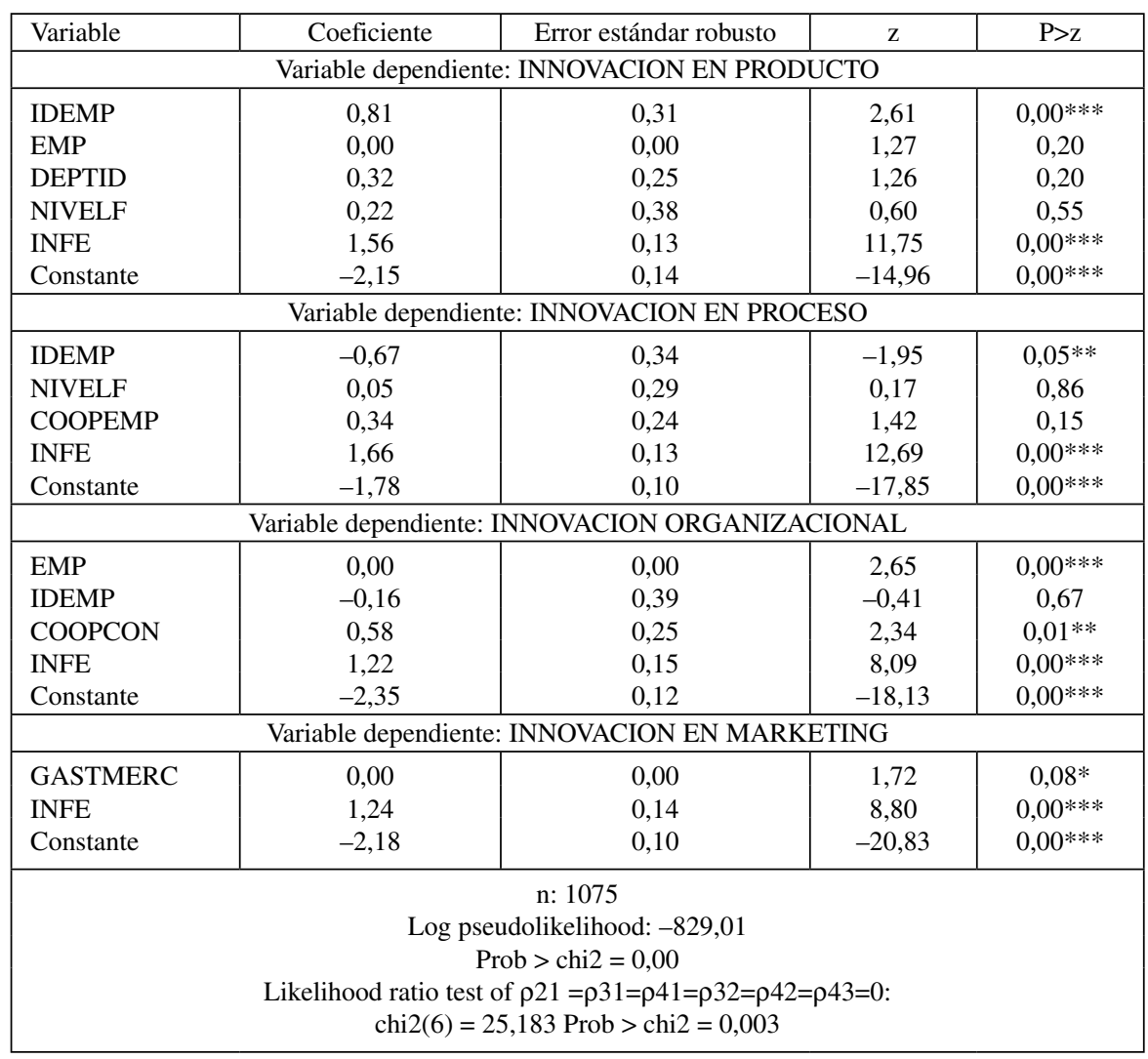

Significancia: $* * * 1 \%, * * 5 \% ; * 10 \%$.

Los resultados corroboran que existe correlación de errores, fundamentalmente cuando se consideran el total de empresas y las Pymes, por tanto la probabilidad de innovación no se podría estimar usando por separado un modelo logit o probit para cada ecuación.

Los modelos aplicados en su conjunto son significativos. En cuanto a las variables específicas, al analizar el total de empresas del sector de alimentos y bebidas se encuentra que el uso de fuentes externas para innovar resulta significativo para explicar una mayor probabilidad de innovar en marketing, procesos y organización. Por su parte, el gasto en I+D y la tenencia de un departamento que coordine estas actividades afecta 


\section{TABLA 6}

ESTIMACION PROBIT MULTIVARIADO EMPRESAS GRANDES DE ALIMENTOS Y BEBIDAS

\begin{tabular}{|c|c|c|c|c|}
\hline Variable & Coeficiente & Error estándar robusto & $\mathrm{z}$ & $\mathrm{P}>\mathrm{Z}$ \\
\hline \multicolumn{5}{|c|}{ Variable dependiente: INNOVACION EN PRODUCTO } \\
\hline IDEMP & 1,48 & 0,31 & 4,66 & $0,00 * * *$ \\
\hline EMP & 0,00 & 0,00 & 2,43 & $0,01 * *$ \\
\hline DEPTID & 0,77 & 0,30 & 2,55 & $0,01 * *$ \\
\hline NIVELF & 0,04 & 0,45 & 0,09 & 0,92 \\
\hline INFE & 1,60 & 0,23 & 6,88 & $0,00 * * *$ \\
\hline Constante & $-2,42$ & 0,22 & $-11,14$ & $0,00 * * *$ \\
\hline \multicolumn{5}{|c|}{ Variable dependiente: INNOVACION EN PROCESO } \\
\hline IDEMP & 0,63 & 0,24 & 2,59 & $0,01 * * *$ \\
\hline NIVELF & 0,20 & 0,40 & 0,49 & 0,62 \\
\hline OBS_ENT & 0,01 & 0,00 & 3,51 & $0,00 * * *$ \\
\hline INFE & 1,52 & 0,23 & 6,72 & $0,00 * * *$ \\
\hline Constante & $-2,12$ & 0,15 & $-14,02$ & $0,00 * * *$ \\
\hline \multicolumn{5}{|c|}{ Variable dependiente: INNOVACION ORGANIZACIONAL } \\
\hline EMP & 0,00 & 0,00 & 0,89 & 0,37 \\
\hline IDEMP & 0,49 & 0,25 & 1,95 & $0,05^{* *}$ \\
\hline INFE & 1,14 & 0,22 & 5,13 & $0,00 * * *$ \\
\hline Constante & $-2,13$ & 0,15 & $-14,35$ & $0,00 * * *$ \\
\hline \multicolumn{5}{|c|}{ Variable dependiente: INNOVACION EN MARKETING } \\
\hline GASTMERC & $8.89 \mathrm{e}-07$ & $5.03 \mathrm{e}-07$ & 1,77 & $0,07 *$ \\
\hline INFE & 1,95 & 0,27 & 7,27 & $0,00 * * *$ \\
\hline Constante & $-2,59$ & 0,24 & $-10,83$ & $0,00 * * *$ \\
\hline \multicolumn{5}{|c|}{$\begin{array}{l}\text { n: } 207 \\
\text { Log pseudolikelihood: }-403,60 \\
\text { Prob }>\text { chi } 2=0,00 \\
\text { ratio test of } \rho 21=\rho 31=\rho 41=\rho 32=\rho 42=\rho 43=0 \text { : } \\
\text { i2 } 2(6)=6,948 \text { Prob }>\text { chi } 2=0,3256\end{array}$} \\
\hline
\end{tabular}

Significancia: $* * * 1 \%, * * 5 \% ; * 10 \%$.

significativamente la obtención de nuevos bienes y servicios. Entretanto, variables como la cooperación con otras empresas, proveedores y competidores (respecto de la innovación en procesos), la cooperación con clientes (sobre la innovación en producto) y la cooperación con fuentes de conocimiento (referidos a la innovación organizacional) también resultan determinantes significativos para innovar.

Analizando solo las Pymes, los tipos de innovación para los que mayor número de variables resultan significativas son la innovación en procesos, la innovación en productos y la innovación organizacional. En el caso de las dos primeras, la realización de inversiones en $\mathrm{I}+\mathrm{D}$ y de actividades de innovación abierta que facilitan el uso de información externa para innovar son factores significativos. A la utilización de 
fuentes externas de información se suma el número de empleados y la cooperación con fuentes generadoras de conocimiento como variables que impactan significativamente la probabilidad de innovar organizacionalmente.

En relación con las empresas grandes, la innovación en producto y procesos son los tipos con mayor cantidad de variables significativas. En las empresas de este tamaño, la mayor inversión en I+D y la presencia de un departamento propio encargado de gestionar esta área, junto con el uso de fuentes externas para innovar, conforman las variables con influencia significativa para la introducción de productos nuevos o mejorados. Para la innovación en procesos, además de los gastos en investigación y desarrollo y el uso de fuentes externas para innovar, el mayor reconocimiento de los obstáculos del entorno incide significativa y positivamente en la probabilidad de innovar. Este hecho sirve de referencia para indicar un avance en el estado de madurez de innovación en las empresas de mayor tamaño del sector, ya que son capaces de identificar sus obstáculos y actuar en pro de superarlos y alcanzar innovaciones en sus procesos.

A partir de los estimativos relativos a la probabilidad marginal de realizar cada tipo de innovación se predijo la probabilidad de participar de cada empresa, tanto para el grupo de tratamiento como de control. Posteriormente se calculó el impacto de realizar cada tipo de innovación en la ejecución de actividades de exportación, como se señala en (1). En este punto se consideraron tres métodos de emparejamiento: vecino más cercano, emparejamiento radial y kernel. El primero es un método en el que se toma a una observación (empresa) del grupo de control con la probabilidad de participación más próxima a la observación tratada.

Asumiendo que no hay empates, el vecino de control más cercano, $C(i)$, del individuo de tratamiento $i$ corresponde a:

$$
C(i)=\left\{j \in T=0\left|\arg \min _{j}\right|\left|P_{i}(X)-P_{j}(X)\right| \mid\right\}
$$

Al no existir limitantes para la distancia entre probabilidades, este método garantiza el emparejamiento entre el grupo de control y el de tratamiento independientemente de su calidad, lo que supone su mayor limitante. Por tal motivo se recurre al método de emparejamiento radial, que define una distancia máxima dentro de la que se realizan los emparejamientos ${ }^{3}$. Su estimador corresponde a:

$$
C(i)=\left\{j \in T=0\left\||| P_{i}(X)-P_{j}(X)\right\| \leq k\right\}
$$

En el caso del PSM por kernel se trata de un estimador no paramétrico que empareja a cada individuo del grupo de tratamiento con un promedio ponderado de

3 Se usó un valor para el radio de 0,1 . 
(potencialmente) todos los individuos del grupo de control. En este estudio se hace uso del kernel Epanechnikov, el que compara a los individuos del grupo de tratamiento con un subconjunto del grupo de control. Formalmente, el impacto promedio en los tratados emparejando bajo el estimador por kernel corresponde a:

$$
\tau_{A T T}^{P S M-\text { Kernel }}=\frac{1}{I}\left\{\sum_{i \epsilon T=1}\left[\left(Y_{i}\right)-\sum_{j \epsilon T=0} w_{i j}\left(Y_{j}\right)\right]\right\}
$$

Donde el impacto viene dado como la diferencia entre la variable resultado de un individuo del grupo de tratamiento, $Y_{i}$, y el promedio ponderado de la variable resultado en el subconjunto de individuos del grupo de control, $Y_{j} . I$ denota los individuos en el grupo de tratamiento y $w_{i j}$ actúa como ponderador asignando los pesos de cada observación del grupo de control. Para el PSM por kernel, el ponderador $w_{i j}$ es una función de qué tan lejos está el individuo $i$ perteneciente al grupo de tratamiento de cada individuo $j$ perteneciente al grupo de control:

$$
w_{i j}^{K E R N E L}=\frac{G\left(\frac{P_{j}(X)-P_{i}(X)}{h}\right)}{\sum_{\substack{k=1 \\ k \in T=0}}^{k} G\left(\frac{P_{j}(X)-P_{i}(X)}{h}\right)}
$$

Donde $G($.$) es una función de kernel, h$ es el ancho de banda y $K$ es la cantidad de individuos en el grupo de control. La función kernel Epanechnikov usada en el trabajo se construye así:

$$
G_{\text {epa }}\left(u_{i}\right)=\left\{\begin{array}{c}
\frac{3}{4}\left(1-u_{i}^{2}\right) \text { si } u_{i}<1 \\
0 \text { si } u_{i} \geq 1
\end{array}\right.
$$

Siendo $u_{i}$ la distancia relativa de cada observación a un punto de referencia.

Los resultados obtenidos se ofrecen en la tabla 7. Como característica distintiva, para los tres métodos de emparejamiento el efecto de realizar innovaciones no tecnológicas sobre la condición exportadora no resulta significativo. Los resultados de mayor interés se encuentran para la innovación en producto y procesos. La estimación kernel revela que para las empresas grandes del sector de alimentos y bebidas, la consecución de nuevos bienes y servicios impacta positiva y significativamente las exportaciones de las firmas. Sin embargo, la magnitud del efecto es marginal. Sorpresivamente, para el caso de las Pymes los emparejamientos kernel y por vecino más cercano indican que innovar en productos impacta negativa y significativamente las ventas en el extranjero para este tipo de compañías. De manera más precisa, una Pyme del sector de alimentos 


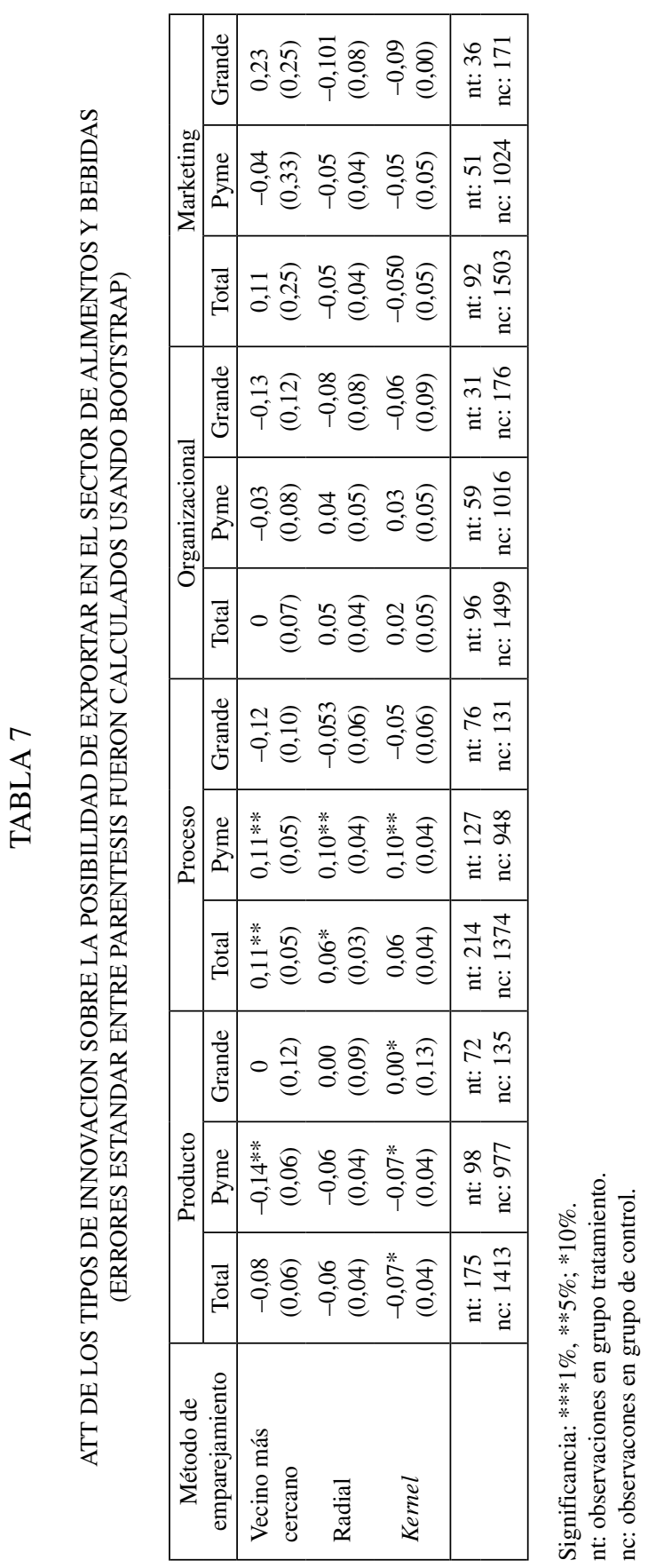


y bebidas que innova en producto tiene una probabilidad promedio de exportar $7 \%$ más baja, bajo el emparejamiento kernel, frente a no adelantar innovación de este tipo. El efecto alcanza el $14 \%$ bajo el método de vecino más cercano.

El impacto de la innovación en procesos resulta esperado. Una Pyme que innova en procesos tiene una probabilidad promedio de exportar $10 \%$ más alta, de acuerdo con los emparejamientos tipos kernel y radial, frente a no realizar este tipo de innovación. La probabilidad se incrementa a $11 \%$ cuando se usa emparejamiento de vecino más cercano.

Estos resultados están en línea con la evidencia presentada, en cuanto sugieren que la innovación tecnológica ejerce la mayor influencia sobre las posibilidades de exportación de una firma; en tanto, la innovación no tecnológica no parece tener un efecto considerable en la condición exportadora.

\subsection{Robustez}

Debido a la naturaleza de sección cruzada de los datos, un posible problema surge como respuesta a la imposibilidad de controlar por efectos fijos inobservables y la aparición de un sesgo escondido, producto de la existencia de variables inobservables que simultáneamente afectan la probabilidad de realizar algún tipo de innovación y la condición de exportar de las empresas. Por estas razones se evaluó la sensibilidad de los resultados a potenciales variables inobservadas.

Para ello se adelantó el test de Mantel-Haenszel (MH), basado en el enfoque de delimitación propuesto por Rosenbaum (2002). En la tabla 8 gamma $(\Gamma)$ es definido en términos de los odds de recibir tratamiento y toma el valor de uno para experimentos aleatorios. Valores elevados de $\Gamma$ significan que el estudio se aleja de un diseño experimental. Entre más alto el nivel de gamma al que el ATT permanece estadísticamente diferente de cero, más robustos son las estimaciones a la potencial influencia del sesgo escondido. El efecto es estadísticamente significativo bajo $\Gamma=1$ y llega a ser aún más significativo para valores crecientes de $\Gamma$ para los que se pudo desestimar el verdadero efecto del tratamiento. El test $\mathrm{MH}$ contempla dos estadísticos, $\mathrm{Q}^{+}$y $\mathrm{Q}^{-}$, que prueban si el efecto del tratamiento es sobreestimado o subestimado, respectivamente. Esto implica que aun si hay un nivel muy alto de heterogeneidad observada, el efecto de realizar algún tipo de innovación sobre la condición exportadora es aún medido por los $p$-values asociados a los estadísticos del test. La tabla 8 presenta los $p$-values para el análisis de sensibilidad de la evaluación de impacto adelantada.

Los resultados se presentan para aquellos casos en los que el ATT fue significativo. Se evidencia que en la mayoría de situaciones el sesgo escondido no impide la existencia de una relación causal entre innovación y la condición exportadora de las empresas del sector de alimentos y bebidas. Sin embargo, para aquellos emparejamientos por kernel para las Pymes que participaron en proyectos de innovación en productos existe sensibilidad cuando se incrementan los odds de innovar. Por ejemplo, si se considera la presencia de una variable inobservable omitida, asociada con un incremento de $10 \%$ 


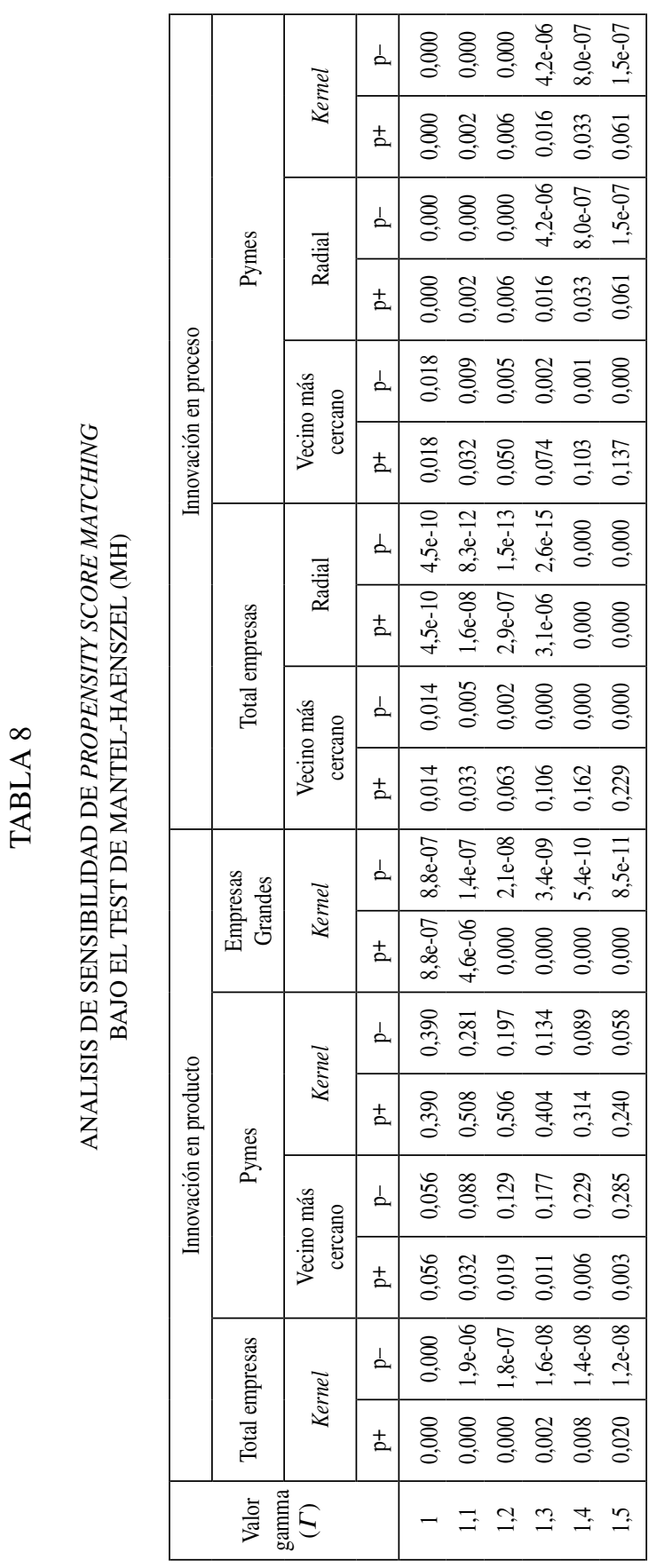


en los odds de innovar en producto $(\Gamma=1,1)$, el $p$-value $\mathrm{p}^{+}$para las Pymes deja de ser significativo, lo que sugiere que los efectos estimados sobreestiman el efecto verdadero de innovar en producto sobre el hecho de exportar en las empresas de este tamaño.

\section{ANALISIS Y DISCUSION}

Los resultados expuestos revelan una clara tendencia para las empresas dedicadas a la producción de alimentos y bebidas de Colombia, en cuanto al impacto de sus actividades de innovación sobre la venta de sus productos en el extranjero. En definitiva, la innovación tecnológica ejerce la mayor influencia sobre las posibilidades de exportación de las empresas del sector. De forma más precisa, la innovación en producto tiene mayor impacto en la promoción de las exportaciones de empresas grandes, mientras que la innovación en procesos lo hace en el caso de las Pymes.

Esto va en línea con lo señalado en la literatura internacional, ya que revalida la idea del efecto positivo de la innovación tecnológica en la internacionalización (Wakelin, 1998), la que es afianzada con los resultados encontrados, en donde se demuestra que la capacidad exportadora de las empresas es mayor cuando existen procesos de innovación en productos o servicios, inversión en I+D y acceso a fuentes externas.

De manera similar, los hallazgos se corresponden con las dinámicas propias del sector, caracterizado por innovaciones de base tecnológica (Samadi, 2014), en su mayoría incrementales en las empresas pequeñas, y radicales en las empresas más grandes (Costa y Jongen, 2006). A pesar de ello, es meritorio hacer algunas precisiones para explicar los resultados.

El hecho que exista una diferencia en el sentido de la dirección del impacto de la innovación en producto entre empresas grandes y Pymes deja entrever posibles disimilitudes en su visión estratégica en torno al desarrollo de nuevos bienes y servicios. De esta forma, para explicar el impacto negativo de este tipo de innovación sobre la conducta exportadora en las Pymes, se sugiere una desconexión entre la concepción de la orientación de mercado y su operacionalización en los mercados internacionales. El punto de partida de esta interpretación recae en la idea que no existe plena identificación entre lo que Diamantopoulos y Hart (1993) llaman compromiso filosófico de la administración en relación con su orientación al mercado y los detalles de acción necesarios para implementarlo en el contexto internacional. En tal sentido, es posible que las Pymes de alimentos y bebidas tengan la disposición de direccionar recursos para adelantar mejoras en las especificaciones técnicas, los componentes y en general en las características funcionales de sus productos, lo que se logra traducir en la introducción de un bien o servicio nuevo o significativamente mejorado. Incluso, es posible que exista un compromiso de exportación por parte de las directivas que los lleve a participar en programas de promoción exportadora, misiones comerciales y recolección de información sobre mercados extranjeros. Sin embargo, probablemente no exista la capacidad de articular estos elementos en una estrategia de exportación 
que apunte a la consolidación de una ventaja competitiva sólida. Precisamente, la evidencia derivada de la EDIT muestra que solo 8,11\% de las Pymes de alimentos y bebidas con innovaciones en producto alcanza los mercados internacionales, reflejando una visión enfocada a innovar para el mercado local.

Al respecto, otro acercamiento a la comprensión del resultado hallado proviene de la posible adopción de estrategias que privilegien la concentración de recursos en segmentos pequeños y específicos del mercado. En la medida que las Pymes de alimentos y bebidas se inclinen a este tipo de estrategias, las innovaciones de productos que lleven a cabo tendrán mayor impacto en el mercado local y no tanto en el internacional. La evidencia muestra que las estrategias de adaptación de productos son capaces de generar incrementos en las ventas pero no mejores resultados en cuanto a mayor participación de mercado (Leonidou, Katsikeas y Samiee, 2002; Calantone et al., 2006). A pesar que una estrategia de adaptación internacional de productos supondría un efecto positivo en la internacionalización (Zucchella y Palamara, 2006), debido a la exigencia y costos que acarrea adaptar productos a las características culturales de consumo de los mercados objetivo, algunas industrias preferirán sacrificar la entrada al exterior para concentrarse en adaptar sus productos a las necesidades de sus consumidores más cercanos y obtener mayores ventas.

Las interpretaciones y explicaciones propuestas requieren mayor profundidad en su análisis y suponen un tema posterior de investigación.

A diferencia de las Pymes, las empresas grandes en esta industria se pueden calificar como de contenido tecnológico medio, caracterizadas por procesos de innovación más complejos que involucran la incorporación de alta tecnología, como la biotecnología, en lugar de la simple adopción de mejoras tecnológicas, por tanto sus productos contienen un valor agregado que favorece la diferenciación de productos y abre el espectro para la comercialización internacional. Este hecho puede explicar en parte el impacto positivo de la innovación en productos sobre las exportaciones en el caso de las empresas de mayor tamaño.

Asimismo, como apuntan Bernard y Jensen (1999), en un conjunto de empresas no exportadoras del mismo sector, aquellas más grandes y productivas tienen más probabilidades de convertirse en exportadoras, debido a que tienen de partida condiciones para superar las barreras que imponen costos adicionales de venta de bienes en los mercados extranjeros, como el establecimiento de un canal de distribución, o gastos de producción para adaptar los modelos nacionales a las exigencias internacionales. Bajo esta perspectiva, la innovación en productos diversificados para distintos mercados geográficos corre mayoritariamente por cuenta de las empresas grandes, mientras que las Pymes tienden a especializarse en los mercados locales.

Por su parte, la evidencia del impacto de la innovación en procesos sobre la condición exportadora muestra que esta forma de innovar tiene incidencia positiva para el grueso del sector de alimentos y bebidas, y resulta significativa para las Pymes. Esto acorde con las características sectoriales, donde las pequeñas y medianas empresas son mayoría y apuntan a competir e ir ganando espacio en el mercado global, a partir de 
la incorporación de nuevos métodos de producción que reducen costos de producción y distribución. También resulta importante la creciente introducción de tecnologías de información que apoyan los procesos auxiliares como la trazabilidad, definitorio en la comercialización internacional.

A esto vale sumar que la innovación en el sector de alimentos y bebidas es principalmente orientada a procesos (Archibugi, Cesarrato y Sirilli, 1991), por lo que las empresas típicas (Pymes primordialmente) introducen cambios sobre la base de tecnologías que provean mejoras operativas y reduzcan costos estructurales. Por su parte, las empresas de mayor tamaño y con capacidad de llevar a cabo inversiones en I+D son más orientadas al producto, por lo que destinan esfuerzos de investigación que generan ventajas competitivas sostenibles sobre el resto de compañías soportadas en la explotación de las nuevas tecnologías y bienes producidos (Traill y Meulenberg, 2002).

Estas diferencias en la orientación estratégica e innovadora resaltan como un elemento sustancial para explicar la heterogeneidad presente en los impactos obtenidos según tamaño empresarial y tipo de innovación.

\section{RECOMENDACIONES DE POLITICA Y CONCLUSIONES}

El entramado empresarial colombiano de la industria de alimentos y bebidas se caracteriza por estar constituido por Pymes; notándose su contribución en la generación de empleo y producción en cada territorio. Su exposición frente al mercado global las hace vulnerables a la hora de competir y, por tanto, la supervivencia de ellas depende en suma medida de la mejora en productividad e innovación, porque actúan como facilitadores para acceder a nuevos mercados, particularmente en el ámbito internacional. Asimismo, existen otras empresas del sector de alimentos y bebidas de gran tamaño, muy significativas por la generación de valor agregado y por integrar cadenas de abastecimiento importantes en la industria colombiana.

A pesar de lo anterior, los resultados muestran que las Pymes y grandes empresas de este sector tienen obstáculos que les impide alcanzar cuotas significativas de mercado internacional. En efecto, las pesquisas demuestran que ocho de cada cien Pymes de alimentos y bebidas que innovan en productos alcanzan los mercados internacionales; lo que hace suponer que son empresas que atienden las necesidades de los consumidores más cercanos mediante bienes tradicionales-artesanales con bajo desarrollo tecnológico, todo ello soportado en la adopción de estrategias focalizadas en la atención de segmentos pequeños y específicos de mercado.

Contrariamente, las grandes empresas en esta industria son de nivel tecnológico medio. En algunos casos, sobresaliendo por sus innovaciones de procesos con una alta incorporación tecnológica, capaces de generar productos con mucho valor agregado, permitiéndole diferenciarse en la comercialización internacional de alimentos y bebidas. Asimismo, se concluye que la gran empresa que no exporta actualmente, tiene mayores posibilidades de penetrar mercados internacionales respecto de la 
mediana, por el hecho de superar más rápidamente las barreras impuestas por los países potencialmente importadores de estos bienes, particularmente los productos que muestren innovación incremental y radical en sus presentaciones.

La evidencia encontrada da cabida a considerar distintas alternativas de intervención de política pública con el fin de guiar la agenda de innovación y, por esta razón, la competitividad del sector de alimentos y bebidas de Colombia. Por las disimilitudes según tamaño empresarial, las políticas a seguir deben tomar un enfoque diferencial. En términos de innovación, particularmente en procesos es donde existe mayor aceptación por la mayoría de las Pymes; quienes adoptan nuevos métodos de producción que buscan disminuir costos de fabricación y distribución. Asimismo, se denota un interés marcado de ofrecer al consumidor un sistema de trazabilidad, soportado sobre las tecnologías de la información y comunicación, lo que garantizaría su penetración en el mercado mundial de alimentos y bebidas.

En consideración de lo anterior, se necesita de mecanismos de reactivación de la tríada empresa - universidad - gobierno, que facilite el financiamiento de infraestructura que estimule las innovaciones de procesos. Por tanto, las futuras estrategias deben dirigirse a la creación o fortalecimiento de espacios multiusos que promuevan el paso de innovaciones de procesos a innovaciones de funciones, donde prime el desarrollo de bienes colectivos con externalidades positivas hacia los empresarios (por ejemplo, desarrollo de imagen de producto, laboratorios de certificación y metrología, entre otros), y de bienes públicos (por ejemplo, plataformas logísticas inteligentes, vías, almacenamiento e institucionalidad de certificación reconocida, entre otros), que interese a los mismos empresarios, academia y centros de investigaciones.

Similarmente, existen aspectos que ameritan mejoras para dinamizar las innovaciones del sector de alimentos y bebidas en Colombia. Por ejemplo, hay varias organizaciones oficiales que promueven conductas innovadoras de forma aislada-las que tienen sus propias políticas y directrices-, ocasionando desarticulación organizacional, lo que se refleja en duplicaciones en el uso de los recursos públicos y privados para los mismos objetivos, desconociéndose los principios económico de la teoría de juego, que nos indica que entre mayor cooperación exista entre agentes, mayores beneficios obtendrían de la alianza. La idea es trazar una estrategia con vínculos de redes horizontales, que permita la creación de confianza, que facilite el entendimiento de las empresas entre sí, y con los demás, evitando los llamados costos de transacción. Como resultado se obtendría un sistema integrado de innovación, que haría posible el establecimiento de consorcios tecnológicos con presencia de empresarios, universidades, centros de investigación, instituciones gremiales y gobierno. Estos consorcios promoverían innovaciones de procesos en el ámbito de la cadena productiva sectorial, destacando los cambios sustantivos de cada empresa, que podrían constituirse en referentes para el resto de la industria.

Considerando que existen brechas en la disponibilidad de recursos económicos entre empresas grandes, medianas y pequeñas, es estratégico seguir apostándole a fondos -alimentados por recursos públicos y privados- que tengan por objeto 
la penetración a nuevos mercados, el financiamiento de esfuerzos más extensos y costosos en $\mathrm{I}+\mathrm{D}$, la conexión con fuentes externas de información, el financiamiento de las innovaciones partiendo de las capacidades empresariales y la protección de la propiedad intelectual. En adición a lo anterior, se hace necesario la implementación de centros de servicios tecnológicos para la industria de alimentos y bebidas, que permita avanzar desde actividades de desarrollo tecnológico menores, hacia actividades de mayores complejidad técnica y sofisticación, y en últimas el acceso a las Pymes a redes de colaboración con centros de investigación o instituciones de educación superior para llevar a cabo iniciativas para el desarrollo tecnológico de productos claves para la industria colombiana de alimentos y bebidas.

Finalmente, para el logro de un mayor dinamismo en la industria de alimentos y bebidas, se requiere de otras acciones gubernamentales relacionadas con el financiamiento de proyectos de riesgo tecnológico, donde se subsidie etapas del proceso innovador (prototipado, protección); consejerías tecnológicas orientadas a sensibilizar a las empresas respecto de sus necesidades y problemas tecnológicos y a encontrar respuesta en la oferta existente en su entorno inmediato.

\section{REFERENCIAS}

AGHION, P.; P. HOWITT.; M. BRANT-COLLETT y C. GARCIA-PEÑALOSA (1998). Endogenous growth theory. MIT press.

ALONSO, J. A. (1994). "El proceso de internacionalización de la empresa", Información comercial española (725), pp. 127-143.

ALVAREZ, R. y R. ROBERTSON (2004). "Exposure to foreign markets and firm-level innovation: Evidence from Chile and Mexico", Journal of international trade and economic development, 13 (1), pp. 57-87.

ARCHIBUGI, D.; S. CESARATTO y G. SIRILLI (1991). "Sources of innovative activities and industrial organization in Italy", Research policy, 20 (4), pp. 299-313.

AVERMAETE, T.; J. VIAENE.; E. MORGAN.; E. PITTS.; N. CRAWFORD y D. MAHON (2004). "Determinants of product and process innovation in small food manufacturing firms", Trends in Food Science \& Technology, 15, pp. 474-483.

AW, B. Y.; M. J. ROBERTS y D. YI XU (2011). "R\&D investment, exporting, and productivity dynamics", The American Economic Review, 101 (4), pp. 1312-1344.

AW, B. Y.; X. CHEN y M. J. ROBERTS (2001). "Firm-level evidence on productivity differentials and turnover in Taiwanese manufacturing". Journal of Development Economics, 66 (1), pp. 51-86.

BALACHANDRA, R. y K. BROCKHOFF (1995). "Are R\&D project termination factors universal?" Research-Technology Management, 38, pp. 31-36.

BALDWIN, J. y Z. LIN (2002). "Impediments to advanced technology adoption for Canadian manufacturers", Research policy, 31 (1), pp. 1-18.

BARNEY, J. (1991). "Firm resources and sustained competitive advantage", Journal of management, 17 (1), pp. 99-120.

BARNEY, J. B. (2001). "Resource-based theories of competitive advantage: A ten-year retrospective on the resource-based view", Journal of management, 27 (6), pp. 643-650.

BERNAL, R. y X. PEÑA (2011). Guía práctica para la evaluación de impacto. Universidad de Los Andes, Facultad de Economía. Ediciones Uniandes, Bogotá.

BERNARD, A. B. y J.B. JENSEN (1999). "Exceptional exporter performance: cause, effect, or both?" Journal of international economics, 47 (1), pp. 1-25. 
BERNARD, A. B.; J. EATON.; J.B. JENSEN y S. KORTUM (2003). "Plants and productivity in international trade", The American Economic Review, 93 (4), pp. 1268-1290.

BHATTACHARYA, M. y H. BLOCH (2004). "Determinants of Innovation", Small Business Economics, 22 (2), pp. 155-162.

BIANCHI, M.; A. CROCE; C. DELL'ERA.; C.A. DI BENEDETTO y F. FRATTINI (2015). “Organizing for inbound open innovation: How external consultants and a dedicated $R \& D$ unit influence product innovation performance", Journal of Product Innovation Management, 33 (4), pp. 492-510.

BOCQUET, R. y P. MUSSO (2010). Product innovation and export performance. En 8th Annual International Industrial Organization Conference.

BOUGRAIN, F. y B. HAUDEVILLE (2002). "Innovation, Collaboration and SMEs Internal Research Capacities", Research Policy, 31 (5), pp. 735-747.

BUSOM, I. (1993). "Los proyectos de I+ D de las empresas: un análisis empírico de algunas de sus características", Revista Española de Economía, pp. 39-65.

BUSTOS, P. (2011). "Trade liberalization, exports, and technology upgrading: Evidence on the impact of MERCOSUR on Argentinian firms", The American economic review, 101 (1), pp. 304-340.

CALANTONE, R. J.; D. KIM; J. B. SCHMIDT y S. T. CAVUSGIL (2006). "The influence of internal and external firm factors on international product adaptation strategy and export performance: a threecountry comparison", Journal of Business Research, 59 (2), pp. 176-185.

CASSIMAN, B.; E. GOLOVKO y E. MARTINEZ-ROS (2010). "Innovation, exports and productivity", International Journal of Industrial Organization, 28 (4), pp. 372-376.

COSTA, A. I. y W. M. F. JONGEN (2006). "New insights into consumer-led food product development", Trends in Food Science \& Technology, 17 (8), pp. 457-465.

COSTANTINI, J. y M. MELITZ (2008). "The dynamics of firm-level adjustment to trade liberalization", The organization of firms in a global economy, 4, pp. 107-141.

CRESPI, G y P. ZUÑIGA (2012). "Innovation and productivity: evidence from six Latin American countries", World development, 40 (2), pp. 273-290.

DE JONG, J. y P. VERMEULEN (2006). "Determinants of Product Innovation in Small Firms", International Small Business Journal, 24 (6), pp. 587-609.

DELGADO, M. A.; J. C. FARINAS y S. RUANO (2002). "Firm productivity and export markets: a nonparametric approach", Journal of international Economics, 57 (2), pp. 397-422.

DIAMANTOPOULOS, A. y S. HART (1993). "Linking market orientation and company performance: preliminary evidence on Kohli and Jaworski's framework", Journal of strategic marketing, 1 (2), pp. 93-121.

EGGERTSSON, T. (1995). El comportamiento económico y las instituciones. Alianza Editorial.

ESTRADA, S. y J. HEIJS (2003). Innovación tecnológica y competitividad: análisis microeconómico de la conducta exportadora en México. Documentos de trabajo del IAIF; $\mathrm{N}^{\circ} 36$.

FREEL, M. (2003). "Sectoral Patterns of Small Firm Innovation, Networking and Proximity". Research Policy, 32 (5), pp. 751-770.

GARCIA, M. y J. BRIZ (2000). "Innovation in the Spanish food \& drink industry". The International Food and Agribusiness Management Review, 3 (2), pp. 155-176.

GORODNICHENKO, Y. y M. SCHNITZER (2010). Financial constraints and innovation: Why poor countries don't catch up. NBER Working Paper 15792.

GOVINDARAJU, V G R C.; G. VIJAYARAGHAVAN y V. PANDIYAN (2013). "Product and process innovation in Malaysian manufacturing: The role of government, organizational innovation and exports". Innovation: Management, Policy \& Practice, 15 (1), pp. 52-68.

GREENAWAY, D. y R. KNELLER (2007). "Firm heterogeneity, exporting and foreign direct investment", The Economic Journal, 117 (517), pp. 134-161.

GROSSMAN, G. M. y E. HELPMAN (1993). Innovation and growth in the global economy. MIT press.

HADJIMANOLIS, A. (2000). "An Investigation of Innovation Antecedents in Small Firms in the Context of a Small Developing Country", $R \& D$ Management, 30 (3), pp. 235-245.

HALLAK, J. C. y J. SIVADASAN (2009). Firms' exporting behavior under quality constraints (No. w14928). National Bureau of Economic Research. 
HALPERN, N. (2010). "Marketing innovation: Sources, capabilities and consequences at airports in Europe's peripheral areas", Journal of Air Transport Management, 16 (2), pp. 52-58.

HARRIS, R. y J. MOFFAT (2011). $R \& D$, innovation and exporting. SERC Discussion paper 73.

HEWITT-DUNDAS, N. (2006). "Resource and capability constraints to innovation in small and large plants", Small Business Economics, 26 (3), pp. 257-277.

HOWELLS, J. (1999). "Research and technology outsourcing", Technology Analysis \& Strategic Management, 11 (1), pp. 17-29.

HOWELLS, J.; D. GAGLIARDI y K. MALIK (2008). “The growth and management of R\&D outsourcing: evidence from UK pharmaceuticals", $R \& d$ Management, 38 (2), pp. 205-219.

HUERGO, E. (2006). The role of technological management as a source of innovation: Evidence from Spanish manufacturing firms, Research Policy, 35 (9), pp. 1377-1388.

JOHANSON, J. y J. E. VAHLNE (2006). "Commitment and opportunity development in the internationalization process: A note on the Uppsala internationalization process model", Management International Review, 46 (2), pp. 165-178.

JOVANOVIC, B. (1982). "Selection and the Evolution of Industry", Econometrica: Journal of the Econometric Society, pp. 649-670.

LAMBARDI, G. y J. MORA (2014). "Determinantes de la innovación en productos o procesos: el caso colombiano", Revista de Economía Institucional, 16 (31), pp. 251-262.

LEONIDOU, L. C.; C. S. KATSIKEAS y S. SAMIEE (2002). "Marketing strategy determinants of export performance: a meta-analysis", Journal of Business research, 55 (1), pp. 51-67.

LOPEZ, J. y R.M. GARCIA (2005). "Technology and export behaviour: a resource-based view approach". International Business Review, 14 (5), pp. 539-557.

MACPHERSON, A. (1997). "A comparison of within-firm and external sources of product innovation", Growth and change 28, pp. 289-308.

MADRID-GUIJARRO, A.; D. GARCÍA y H. VAN AUKEN (2010). "Barriers to Innovation among Spanish Manufacturing SMEs", Journal of Small Business Management, 47 (4), pp. 465-488.

MAIRESSE, J. y P. MOHNEN (2005). "The Importance of R\&D for innovation: a reassessment using French survey data", Journal of Technology Transfer, 30 (1/2), pp. 183-197.

MARQUEZ-RAMOS, L.; I. MARTINEZ-ZARZOSO y C. SUAREZ-BURGUET (2011). "Determinants of deep integration: examining socio-political factors", Open Economies Review, 22 (3), pp. 479-500.

MARTINS, I.; E. GOMEZ-ARAUJO y Y. VAILLANT (2014). "The effect of export behavior on ownermanager's commitment to innovation in Colombia: insights from learning approach", Cuadernos de Administración, 27 (49), pp. 135-153.

MEDRANO, N. (2011). Factores determinantes de la adopción de innovaciones en marketing: empresa y comercio (Tesis de Maestría). Facultad de Ciencias Empresariales, Universidad de la Rioja.

MELITZ, M. (2003). "The impact of trade on intra-industry reallocations and aggregate industry productivity", Econometrica, 71 (6), pp. 1695-1725.

MILESI, D. y C. AGGIO (2008). "Éxito exportador, innovación e impacto social. Un estudio exploratorio de PYMES exportadoras latinoamericanas", FUNDES/BID.

MOORI KOENIG, V.; D. MILESI, y G. YOGUEL (Ed.). (2001). Ventajas competitivas dinámicas: las PYMES exportadoras exitosas argentinas. Editorial Miño y Dávila, Buenos Aires.

MOORI KOENIG, V.; G. RODRIGUEZ; G. YOGUEL y A. GRANADOS (2005). Perfil de la PYME exportadora exitosa, el caso colombiano. Ventajas competitivas dinámicas: las PYMES exportadoras exitosas argentinas. FUNDEs, Bogotá, Colombia.

MOORI KOENIG, V.; G. YOGUEL.; D. MILESI.; y I. GUTIERREZ (2004). Buenas prácticas de las PYME exportadoras exitosas: el caso chileno. FUNDEs, Santiago, Chile.

NASSIMBENI, G. (2001). "Technology, innovation capacity, and the export attitude of small manufacturing firms: a logit/tobit model”, Research Policy, 30 (2), pp. 245-262.

NOCKE, V. y S. YEAPLE (2006). Globalization and endogenous firm scope (No. w12322). National Bureau of Economic Research.

OECD. (2005). Manual de Oslo. Guía para la recolección e interpretación de datos de innovación (3ra ed). OECD Publications, París. 
PALANGKARAYA, A. (2012). "The link between innovation and export: Evidence from Australia's small and medium enterprises", ERIA Discussion Paper, 8.

PAVITT, K. (1984). "Sectoral patterns of technical change: towards a taxonomy and a theory", Research policy, 13 (6), pp. 343-373.

PEDRERO, Y. (2014). La estrategia de internacionalización: análisis comparativo de los mecanismos de entrada en mercados exteriores de seis empresas que operan en diferentes sectores de la economía (Tesis de maestría). Facultad de Economía y Empresa. Universidad de Barcelona. Recuperado de http://diposit.ub.edu/dspace/bitstream/2445/66261/1/TFM_MOI_Pedrero-Yolanda-jun2015.pdf

PENROSE, E.T. (1959). The Theory of the Growth of the Firm. Blackwell, Oxford.

PERIS, M. L. F.; M. J. O. MESTRE y C.C. ZORNOZA (2004). "El efecto de la estrategia de innovación tecnológica y de la estrategia exportadora en el desempeño internacional de la empresa", Cuadernos de Economía y Dirección de la Empresa, (20), pp. 151-174.

REICHSTEIN, T. y A. SALTER (2006). "Investigating the sources of process innovation among UK manufacturing firms", Industrial and Corporate Change, 15 (4), pp. 653-682.

RODIL, O.; X. VENCE y M. SANCHEZ (2015). "The relationship between innovation and export behaviour: The case of Galician firms". Technological Forecasting and Social Change.

ROGERS, M. (2004). "Networks, Firm Size and Innovation", Small Business Economics, 22 (2), pp. 141-153.

ROMIJN, H. y M. ALBALADEJO (2002). "Determinants of Innovation Capability in Small Electronics and Software Firms in Southeast England", Research Policy, 31 (7), pp. 1053-1067.

ROPER, S. y J. H. LOVE (2002). "Innovation and export performance: evidence from the UK and German manufacturing plants", Research policy, 31 (7), pp. 1087-1102.

ROSENBAUM, P. R. (2002). Observational studies. En Observational Studies (pp. 1-17). New York: Springer.

ROSENBAUM, P. R. y D. B. RUBIN (1983). "The central role of the propensity score in observational studies for causal effects", Biometrika, 70 (1), pp. 41-55.

ROUVINEN, P. (2002). "Characteristics of product and process innovators: some evidence from the Finnish innovation survey", Applied Economics Letters, 9 (9), pp. 575-580.

SALOMON, R. M. y J. M. SHAVER (2005). "Learning by exporting: new insights from examining firm innovation”, Journal of Economics \& Management Strategy, 14 (2), pp. 431-460.

SAMADI, S. (2014). "Open innovation business model in the food industry: Exploring the link with academia and SMEs", Journal of Economics, Business and Management, 2 (3), pp. 209-213.

SAPPRASERT, K. (2008). On factors explaining organisational innovation and its effects (No. 20080601). TIK Working Papers on Innovation Studies.

TRAILL, B. y K. G. GRUNERT (ed) (1997). Product and process innovation in the food industry, Blackie Academic and Professional, Londres.

VAN BEVEREN, I. y H. VANDENBUSSCHE (2009). Exports, innovation and productivity: Firm-level evidence for Belgium. En Proceedings of the 11th ETSG conference (online). University of Rome 'Tor Vergata'. Faculty of Economics.

VERHAEGHE, A. y R. KFIR, (2002). "Managing innovation in a knowledge intensive technology organization (KITO)", $R \& D$ Management, 32, pp. 409-417.

WAKELIN, K. (1998). "Innovation and export behaviour at the firm level". Research policy, 26 (7), pp. 829-841.

YEAPLE, S. R. (2005). “A simple model of firm heterogeneity, international trade, and wages", Journal of international Economics, 65 (1), pp. 1-20.

YOON, E. y G. L. LILIEN (1985). "A new product launch-time decision model”, Journal of Product Innovation Management, 3, pp. 134-144.

ZUCCHELLA, A. y G. PALAMARA (2006). "Niche strategy and export performance", Advances in international marketing, 17 (1), pp. 63-87. 


\section{ANEXOS}

\section{TABLA A1}

ESTADISTICAS DESCRIPTIVAS VARIABLES MODELO PROBIT MULTIVARIADO. TOTAL EMPRESAS DE ALIMENTOS Y BEBIDAS

\begin{tabular}{|l|r|r|r|r|}
\hline \multicolumn{1}{|c|}{ Variables } & Media & $\begin{array}{c}\text { Desviación } \\
\text { estándar }\end{array}$ & Máximo & Mínimo \\
\hline IDEMP (Miles de pesos & 53,78 & 845,01 & 30422,54 & 0 \\
colombianos) & 0,05 & 0,23 & 1 & 0 \\
DEPTID & 0,24 & 0,2 & 100 & 0 \\
NIVELF & 0,07 & 0,25 & 1 & 0 \\
COOPEMP & 0,03 & 0,19 & 1 & 0 \\
COOPCLI & 0,06 & 0,23 & 1 & 0 \\
COOPCON & 0,2 & 0,4 & 1 & 0 \\
INFE & 11790,06 & 170096,6 & 4041523 & 0 \\
GASTMERC (Miles de pesos & 132,52 & 390,48 & 6153 & 0 \\
colombianos) & \multicolumn{3}{|r}{} \\
EMP &
\end{tabular}

TABLA A2

ESTADISTICAS DESCRIPTIVAS VARIABLES MODELO PROBIT MULTIVARIADO. PYMES ALIMENTOS Y BEBIDAS

\begin{tabular}{|l|r|r|r|r|}
\hline \multicolumn{1}{|c|}{ Variables } & Media & $\begin{array}{c}\text { Desviación } \\
\text { estándar }\end{array}$ & \multicolumn{1}{c|}{ Máximo } & Mínimo \\
\hline IDEMP (Miles de pesos & 46,7 & 973,22 & 30422,54 & 0 \\
colombianos) & 0,03 & 0,17 & 1 & 0 \\
DEPTID & 0,22 & 0,18 & 100 & 0 \\
NIVELF & 0,04 & 0,21 & 1 & 0 \\
COOPEMP & 0,03 & 0,18 & 1 & 0 \\
COOPCON & 0,18 & 0,39 & 1 & 0 \\
INFE & 2160,857 & 57810,46 & 1888411 & 0 \\
GASTMERC (Miles de pesos & 50,16 & 43,18 & 199 & 11 \\
colombianos) & & & \\
EMP & &
\end{tabular}


TABLA A3

ESTADISTICAS DESCRIPTIVAS VARIABLES MODELO PROBIT MULTIVARIADO. EMPRESAS GRANDES DE ALIMENTOS Y BEBIDAS

\begin{tabular}{|l|r|r|r|r|}
\hline \multicolumn{1}{|c|}{ Variables } & Media & $\begin{array}{c}\text { Desviación } \\
\text { estándar }\end{array}$ & Máximo & Mínimo \\
\hline IDEMP (Miles de pesos & 162,03 & 748,41 & 8400,18 & 0 \\
colombianos) & 0,27 & 0,44 & 1 & 0 \\
DEPTID & 0,3 & 0,18 & 94,8 & 1,8 \\
NIVELF & 750,62 & 852,5 & 6153 & 201 \\
EMP & 40,09 & 42,01 & 100 & 0 \\
OBS_ENT & 0,52 & 0,5 & 1 & 0 \\
INFE & 79595,27 & 448486,2 & 4041523 & 0 \\
GASTMERC (Miles de pesos & & & & \\
colombianos) & & &
\end{tabular}

